\section{Potential downsides of perfect pain relief}

SIR - J. J. Cox and colleagues present an exciting aspect of using drugs targeting SCN9A as analgesic agents, in their Article "An SCN9A channelopathy causes congenital inability to experience pain" (Nature 444, 894-898; 2006). As clinicians, we need better tools to treat the high prevalence of nonmalignant chronic pain syndromes such as back pain, arthritis, fibromyalgia, migraines and irritable bowel syndrome. These afflict a large number of people and severely reduce their quality of life, as well as creating a vast financial burden.

Several novel analgesics, targeting painspecific molecular transduction pathways, are at various levels of clinical and preclinical development. The high drug specificity potentially allows profound pain relief without the dose-limiting toxicities seen with current drugs. But what if these medicines are even better than expected?

Pain is a protection mechanism, serving to prevent injury, deter repetitive harmful behaviour and allow healing. It is usually the first indication of disease or trauma that triggers the individual to seek treatment. Our current approaches to treating pain often provide effective relief, but it is 'incomplete', in that activity-related breakthrough pain continues - and this can be useful.

The problem will arise with long-term use of a potentially perfect analgesic drug to alleviate a specific symptom, such as chronic back pain. The medications that block the targeted pain generator could also prevent the warning pain caused by a new, more serious condition such as stroke, myocardial infarction or bowel obstruction. The results could be catastrophic: diagnosis may be delayed, leading to irreversible damage, long-term disability or worse.

Using a perfect analgesic to relieve pain from sports injuries such as sprain, repetitive injury or overuse may allow the athlete to ignore the bio-warning signs of pain, impair the healing process and risk more serious injury.

Should we be deterred from the search for superior pain medications? In patients with advanced life-threatening disease seeking palliation, the question is easily answered: no. But a young patient with disabling lowerback pain on chronic medication presents a different situation. Relieving the back pain may restore function, but could hinder the rapid diagnosis of life-threatening disease.

Our choice is whether to limit the use of these medications to a defined population, on the basis of risk assessment, or to embrace these innovations and attempt to adjust the practice of medicine fast enough to accommodate the potential consequences. In the latter case, clinicians would have to remove 'pain' from the list of symptoms currently used in diagnosis. For instance, myocardial infarction may present with more flu-like symptoms - sudden onset of nausea, weakness, diaphoresis and shortness of breath, instead of the classically described patient clutching his or her chest. Appendicitis and bowel perforation may present with painless nausea, vomiting and loss of appetite.

On the other hand, denying a known therapy to a patient with unrelenting pain is difficult to accept, when a novel analgesic could normalize function, improve quality of life, reduce the financial impact by replacing ineffective treatments and allow an earlier return to work.

The dilemma outlined here, although theoretical, should be considered before these therapies are approved. If these much-needed therapeutic advances are to be used in a truly effective fashion, anticipating and preventing any possible untoward events could facilitate their safe implementation. Data collected in clinical trials would be helpful in determining the importance of this response.

Andrew Mannes ${ }^{\star}$, Michael ladarola $\uparrow$

*Department of Anesthesia and Surgical Services, Warren Grant Magnuson Clinical Center, National Institutes of Health, 10 Center Drive, Bethesda, Maryland 20892, USA

$\uparrow$ Neurobiology and Pain Therapeutics Section, National Institute of Dental and Craniofacial Research, 49 Convent Drive, MSC 4410, Bethesda, Maryland 20892, USA

\section{Need to distinguish science (good or bad) from ethics}

SIR - Although I share John Horgan's concern about the misrepresentation of science by the current US administration and others, expressed in his Book Review of Seth Shulman's Undermining Science ("Dark days at the White House" Nature 445, 365-366; 2007), he and other commentators need to distinguish clearly between science and ethics in their arguments. It is bad science to claim that reducing environmental protection will not have adverse effects on rare species, for example, but the decision whether we should protect rare species or not is an ethical one.

With regard to research on embryonic stem cells, I know of no one who denies that there would be at least some scientific insights and medical benefit from such research. However, the real question with regard to stem-cell research is whether the potential medical benefit and scientific knowledge outweigh any harm done to the embryo. The answer depends strongly on the value assigned to the embryo, which is not a scientific question. Thus, instead of being an example of science versus anti-science, this is a case of competing ethical claims.
Replacing an advocate of stem-cell research on the President's Council on Bioethics with someone morally opposed to it reflects support for an ethical position (although the fact that this particular example involved replacing a biologist with a political philosopher does also raise the possibility that science was getting less say).

By invoking science as supporting a particular position on ethical questions, which science cannot directly answer, critics are making an error of logic similar to the one made by the Bush administration itself.

David Campbell

Department of Biological Sciences, 425 Scientific Collections Building, University of Alabama, Box 870345, Tuscaloosa, Alabama 35487-0345, USA

\section{Never mind the footprint, get the mass right}

SIR - Your correspondent Geoffrey Hammond is being somewhat pedantic in calling for the 'carbon footprint' - amounts of carbon expressed in tonnes - to be called instead the 'carbon weight', because the essential property of a footprint is its area ("Time to give due weight to the 'carbon footprint' issue" Nature 445, 256; 2007).

A true pedant would, however, have gone the full distance! If units of carbon are expressed in kilograms, tonnes and so on, the proper term is 'carbon mass'. Weight is the vertical force acting on a body as a result of gravity. The SI unit of weight is a newton, which has base dimensions of $\mathrm{kg} \mathrm{m} \mathrm{s}^{-2}$. Balances measure weight, but are calibrated to give the answer in units of mass.

'Footprint' has become an accepted term throughout the ecological and environmental sciences, irrespective of dimensions and units, such as my domestic carbon footprint (tonnes) or my flux-tower carbon footprint (metre radius or hectares). 'Footprint' is evocative and has meaning, with relevant and appropriate units.

A far more serious issue is that journalists and commentators frequently confuse the mass of carbon and the mass of $\mathrm{CO}_{2}$, sometimes within the same article. The mass of a mole of $\mathrm{CO}_{2}$ is almost 4 times (44/12) larger than the mass of a mole of carbon. With errors of this size in articles and reports, it is not surprising that the public at large is confused by apparently conflicting reports on atmospheric $\mathrm{CO}_{2}$ concentrations and terrestrial carbon budgets. Absolute clarity and consistency in definition is essential. Paul Jarvis

Institute of Atmospheric and Environmental Science, School of Geosciences, University of Edinburgh, Edinburgh EH9 3JN, UK

Contributions to Correspondence may be submitted to corres@nature.com. 